\title{
Dynamique des populations fongiques et bactériennes de la rhizosphère des noisetiers truffiers. II. Chélation du fer et répartition taxonomique chez les Pseudomonas fluorescents
}

\author{
M. Mamoun et J.M. Olivier \\ INRA, Station de recherches sur les champignons, Centre de recherches de Bordeaux, Villenave-d'Ornon, 33140 Pont-de-la-Maye, \\ France
}

(reçu le 27-7-1988, accepté le 22-12-1988)

Résumé - Pour préciser l'environnement de Tuber melanosporum artificiellement associée au noisetier, les populations bactériennes des sols de truffières ont été analysées de façon quantitative (dénombrement) et qualitative (aptitude à chélater le fer et identification taxonomique). Les Pseudomonas fluorescents sont surtout actifs au niveau du rhizoplan où l'espèce $P$. fluorescens sensu stricto représente le taxon principal. Un groupe taxonomique intermédiaire entre $P$. fluorescens et $P$. putida s'est révélé caractéristique de la zone racinaire. Les germes ayant les plus fortes aptitudes à chélater le fer sont également localisés dans la proche rhizosphère. La discussion porte sur la valeur écologique des résultats dans le cas particulier des sols calcaires de truffières fortement chlorosants et intègre les conséquences microbiologiques de variations du potentiel hydrique précédemment mises en évidence. Enfin, les hypothèses concernant le rôle des bactéries du sol sur la pérennité d'une mycorhization artificielle sont avancées.

truffe - Tuber - bactéries - Pseudomonas fluorescens - P. putida - hydroxy-8-quinoléine - fer - chlorose potentiel hydrique

\begin{abstract}
Summary - Dynamics of fungal and bacterial populations in the rhizosphere of truffle producing hazelnut trees. II. Iron chelation and taxonomic distribution in fluorescent Pseudomonads populations. Bacterial populations were analysed to determine the biological interactions in the rhizosphere of hazelnut trees artificially mycorrhized with Tuber melanosporum. Studies were carried out by selective countage (quantitative analysis), taxonomical identification and bacterial iron chelating properties (qualitative analysis). Fluorescent Pseudomonads, and especially P. fluorescens sensu stricto are dominant on the rhizoplane. $A$ taxonomic group intermediate between $P$. fluorescens and $P$. putida was showed to be characteristic of the rhizoplane. The more chelating powerfull strains are also closely related to the mycorrhized roots surface. Discussion is based on the ecological value of such results in the particular situation of strongly chlorotic calcareous soils of truffle areas. Previous results on the microbiological effects of water potential variations are also considered. Hypothesis concerning the incidence of soil bacteria on the perenniality of the artificial mycorrhization are finally proposed.
\end{abstract}

truffle - Tuber - bacteria - P. fluorescens - P. putida - 8-hydroxyquinoline - iron - chlorotic soil - matric potential

\section{Introduction}

L'étude microbiologique des sols de truffières a été entreprise pour identifier les facteurs conditionnant la pérennité et l'extension de la symbiose ectomycorhizienne entre la truffe noire Tuber melanosporum et la plante hôte.
Un antagonisme entre Pseudomonas et mycélium de Tuber a été observé in vitro (Mamoun et al., 1985). Puis une corrélation étroite entre les effectifs de Pseudomonas fluorescents et la teneur en eau du sol a été mise en évidence au niveau du rhizoplan de noisetiers truffiers, alors qu'elle n'existe pas dans le sol nu (Olivier et 
Mamoun, 1988). La colonisation bactérienne du rhizoplan est à son maximum en période hivernale, à son minimum en période estivale lorsque l'activité mycélienne de la truffe s'intensifie. L'irrigation de la truffière peut perturber cet équilibre naturel. En effet, les observations "au champ" de Le Tacon et al. (1982) et Verlhac (communication personnelle) montrent qu'une irrigation modérée a un effet bénéfique, tandis que des apports importants entraînent un arrêt de la production. II semble donc qu'une teneur en eau excessive (ou au moins au delà d'un certain seuil) soit favorable à certaines bactéries et dépressive pour le champignon. En sols de truffières, calcaires et fortement chorosants, le statut hydrique n'est pas le seul paramètre pouvant intervenir dans la compétition entre Pseudomonas et Tuber. Poitou et Cassin (1987) ont observé une teneur en fer élevée dans des ascocarpes de Tuber melanosporum (100 à 300 ppm). La production de sidérophores a été mise en évidence chez les champignons ectomycorhiziens (Szanislo et al., 1981) et chez les Pseudomonas fluorescents (Hohnadel et Meyer, 1986; Scher, 1986). Kloepper et al. (1980) attribuent à ces sidérophores un rôle d'intermédiaire dans la mobilisation du fer dans la rhizosphère.

En conditions de carence en fer assimilable, comme c'est le cas dans les truffières, on peut envisager l'hypothèse d'une compétition pour cet élément entre Pseudomonas et Tuber, le potentiel hydrique influençant l'équilibre $\mathrm{Fe} \| \mathrm{I} / \mathrm{Fe}$ III (Lindsay, 1974). Dans le présent travail, le pouvoir complexant des Pseudomonas est quantifié selon leur site d'isolement (rhizoplan, rhizosphère et sol nu) et selon leur rattachement taxonomique.

Des bactéries autres que les Pseudomonas produisant des sidérophores (Byers, 1986)), les germes non fluorescents, sont également prises en considération.

\section{Matériel et méthodes}

Les isolements bactériens sont effectués au mois de décembre, période d'intense activité des Pseudomonas (Olivier et Mamoun, 1988), à partir d'échantillons prélevés sur la truffière expérimentale INRA de Coulaures (Dordogne). Pour chaque site de prélèvement (rhizoplan, rhizophère et sol nu) deux collections de micro-organismes sont constituées, l'une regroupant des Pseudomonas fluorescents, l'autre des germes non fluorescents. Les isolats sont prélevés à partir des étalements de suspensions-dilutions de sol en boîtes de Petri selon la méthode décrite par Olivier et Guillaumes, (1976).

Un premier tri est effectué d'après l'aspect macroscopique des colonies, selon la détermination de Samson (communication personnelle), de manière à sélec- tionner un ensemble de germes représentatif des différents phénotypes observés dans chaque site.

L'isolement et la purification des isolats se font sur milieu B de King (King et al., 1954) permettant en outre de vérifier pour chacun d'entre eux le caractère de fluorescence.

Les collections obtenues se composent respectivement, pour les Pseudomonas, de 95 isolats provenant du rhizoplan, 93 de la rhizosphère, et 47 du sol nu, et pour les germes non fluorescents, de 44 isolats récoltés sur le rhizoplan, 38 dans la rhizosphère et 29 dans le sol nu.

Détermination des capacités chélatantes vis-àvis du fer

Le pouvoir chélatant des isolats est déterminé selon la technique adaptée par Lemanceau (1988). La croissance bactérienne est mesurée sur le milieu de Meyer et Abdallah (1978) en présence de doses croissantes d'un ligand de synthèse: l'hydroxy-8-quinoléine, complexant préférentiellement le fer dans les conditions expérimentales décrites (Lemanceau et al., 1988).

Les cultures sont conduites en plaques de microtitration. Chaque puit reçoit $150 \mu$ de substrat nutritif et $50 \mu l$ d'une suspension bactérienne (environ 107 germes $/ \mathrm{ml}$ ) obtenue à partir d'une culture âgée de $24 \mathrm{~h}$. La gamme de concentrations d'hydroxy-8-quinoléine utilisée correspond à des apports de 0-2-4-8-1632 et $64 \mathrm{ppm}$ dans le milieu final. De l'eau "ultra pure" (milli-Q®Millipore) est utilisée pour la confection du milieu de culture et la mise en suspension des bactéries. Chaque isolat est apporté dans 4 puits par milieu, et l'expérience est renouvelée 3 fois.

Le développement bactérien est évalué, après 4 jours d'incubation, par mesure de la turbidimétrie à $620 \mathrm{~nm}$ (photomètre Titertek Multiscan MCC). La concentration minimale inhibitrice (CMI) d'hydroxy-8quinoléine correspond au plus faible apport de ligand pour lequel la valeur obtenue est inférieure à un seuil fixé à 0,1 unité de densité optique (DO).

Les résultats sont exprimés, pour chaque classe de CMI, soit en pourcentages (PI), soit en logarithme des effectifs calculés $(E)$, ces derniers intégrant l'effectif total dénombré précédemment pour le site étudié (Olivier et Mamoun, 1988).

$\mathrm{PI}(\%)=\mathrm{e} \times 100 / \mathrm{n}$ où $\mathrm{e}=$ effectif bactérien pour une classe donnée et $n=$ nombre total d'isolats dans la collection;

$E(C F U / g ~ M S)=P I \times E T$ où $E T=$ effectif total dénombré (en CFU/gMS) à la date d'isolement et CFU = nombre d'unités donnant naissance à une colonie.

Pour chaque représentation graphique les valeurs moyennes sont indiquées, accompagnées de leurs écarts types.

Les comparaisons entre sites de prélèvements sont effectuées selon le test $2 i$ d'après Sokal et Rohlf (1969). Les valeurs indiquées pour $P$ donnent la probabilité d'avoir 2 distributions identiques.

\section{Taxonomie}

Les Pseudomonas fluorescents sont classés au sein des espèces fluorescens et putida, et des deux groupes intermédiaires définis selon Palleroni (1984). Ce classement est basé sur la mise en évidence de deux aptitudes métaboliques (la protéolyse et l'utilisa- 
tion du tréhalose). Les deux sont présentes chez $P$. fluorescens et absentes chez $P$. putida, alors que l'une ou l'autre seulement s'exprime dans les groupes intermédiaires.

Les groupes intermédiaires sont nommés respectivement: i1 pour les bactéries utilisant le tréhalose et non protéolytiques, i2 pour les isolats protéolytiques, mais n'utilisant pas le tréhalose.

\section{Protéolyse}

Les Pseudomonas sont ensemencés par traits sur un milieu de culture gélosé contenant de la gélatine (Luisetti et al., 1972). Après incubation à $25^{\circ} \mathrm{C}$ pendant 4 jours, l'ajout de réactif de Frazier (1926) met en évidence les colonies protéolytiques produisant un halo clair.

\section{Utilisation du tréhalose}

Une solution de tréhalose (stérilisée par filtration sur membrane Millipore $(\circledR) 0,22 \mu \mathrm{m}$ ) est apportée au milieu de Ayers et al. (1919) à la concentration finale en carbone de $0,3 \%$. Le substrat est réparti en plaques de microtitration à raison de $150 \mu \mathrm{l}$ par puit. Chaque cupule reçoit $50 \mu \mathrm{l}$ d'une suspension bactérienne effectuée à partir d'une culture âgée de $24 \mathrm{~h}$ (environ 107 germes $/ \mathrm{ml}$ ). Chaque isolat est apporté dans 4 puits, et l'expérience est renouvelée 3 fois.

Le développement des Pseudomonas est mis en évidence par le virage de l'indicateur de $\mathrm{pH}$ (bleu de bromothymol) contenu dans le milieu de culture. Les notations sont effectuées pendant 3 semaines.

\section{Résultats}

La détermination du pouvoir chélatant à l'intérieur de chaque collection de bactéries montre que, chez les Pseudomonas fluorescents (Fig. 1) les distributions, quoique centrées sur les mêmes CMI (8-16ppm) pour les 3 sites d'isolement, diffèrent significativement entre elles $(0,001<P \leq 0,01)$. Le pourcentage de germes ayant les plus fortes capacités chélatantes (CMl>64ppm) diminue si on s'éloigne de la racine.

Les bactéries non fluorescentes (Fig. 2) présentent également des distributions significativement différentes $(P<0,001)$ selon le site d'isole-

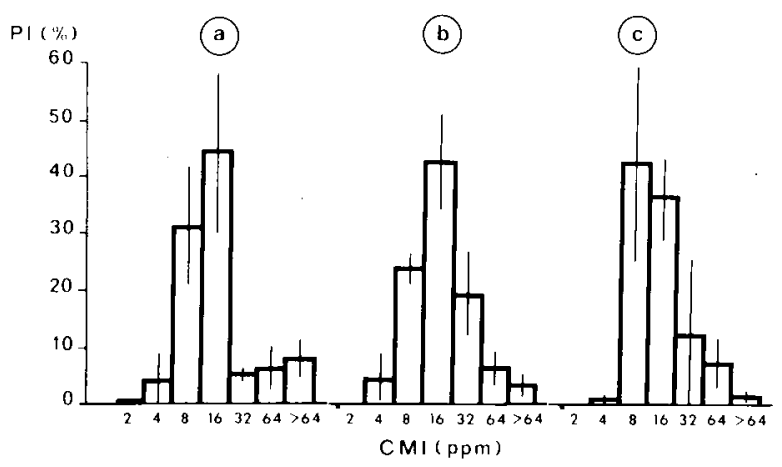

Fig. 1. Pouvoir chélatant des Pseudomonas selon le site d'isolement. Résultats exprimés en \% des isolats (PI) pour chaque concentration inhibitrice ( $\mathrm{CMI}$ ) d'hydroxy-8-quinoléine. a rhizoplan, b rhizosphère, $c$ sol nu.

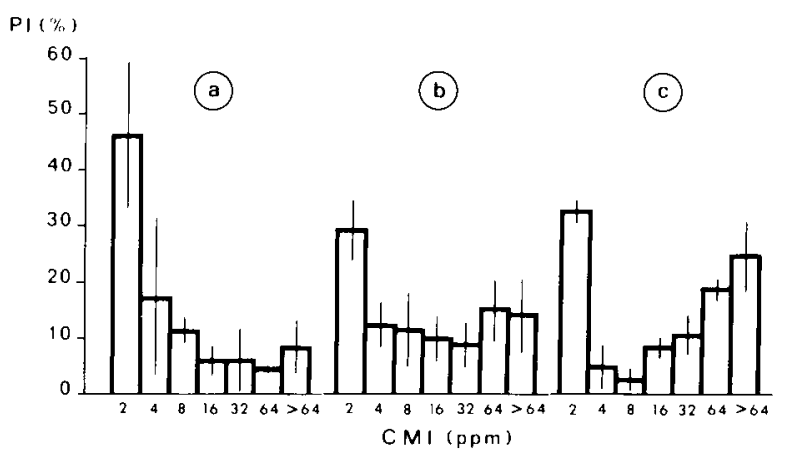

Fig. 2. Pouvoir chélatant des bactéries non fluorescentes selon le site d'isolement. Résultats exprimés en \% des isolats (PI) pour chaque concentration inhibitrice (CMI) d'hydroxy-8quinoléine. a rhizoplan, b rhizosphère, $c$ sol nu.

ment. Les isolats à faible pouvoir chélatant (CMI $=2$ ppm) dominent sur le rhizoplan. Cette dominance s'atténue dans la rhizosphère avec une tendance à une répartition équilibrée entre les différentes classes. Dans le sol nu, la distribution devient bimodale, le pourcentage de germes diminue au niveau des classes moyennes, puis augmente progressivement et de manière importante aux plus fortes CMI.

Les effectifs calculés à partir des résultats précédents font apparaître, pour les Pseudomonas du rhizoplan, un pouvoir chélatant global nettement supérieur à celui détecté dans le sol nu, avec une différence particulièrement marquée pour la plus forte CMI (Fig. 3). Les germes de la rhizosphère ont un comportement intermédiaire.

Les bactéries non fluorescentes à faible ou moyenne capacité chélatante sont davantage représentées au niveau du rhizoplan (Fig. 4). Par contre aucune différence liée au site d'isolement

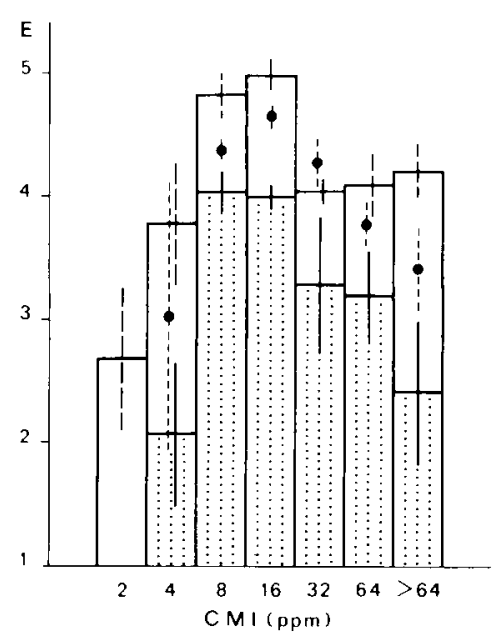

Fig. 3. Répartition du pouvoir chélatant des Pseudomonas dans les 3 sites d'isolement. Résultats exprimés en effectifs calculé $(E=\log \mathrm{CFU} / \mathrm{g} \mathrm{MS})$ pour chaque concentration inhibitrice (CMI) d'hydroxy-8-quinoléine. '1) rhizoplan, rhizosphère, 電 sol nu. 


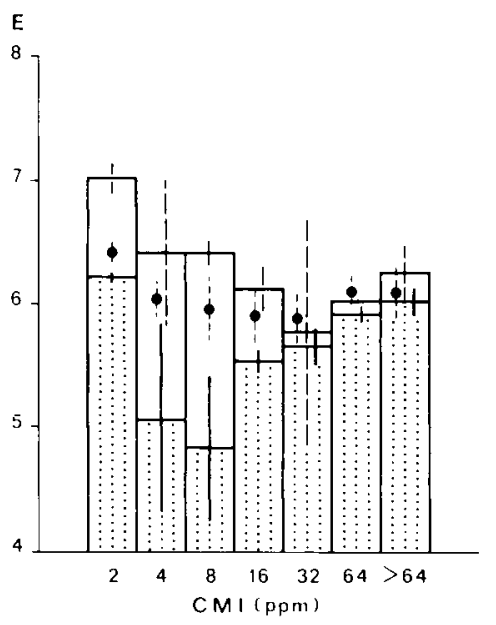

Fig. 4. Répartition du pouvoir chélatant des bactéries non fluorescentes dans les 3 sites d'isolement. Résultats exprimés en effectifs calculé ( $E=\log C F U / g M S$ ) pour chaque concentration inhibitrice (CMI) d'hydroxy-8-quinoléine (cf. Fig. 3.).

n'est observée pour les CMI les plus élevées ( $\geq 32 \mathrm{ppm}$ ).

Le classement taxonomique des Pseudomonas montre que l'espèce $P$. fluorescens constitue l'essentiel de la population isolée, quelle que soit la zone de prélèvement (Fig. 5). Le groupe intermédiaire i2, représentant 20 à $25 \%$ des isolats provenant du rhizoplan et de la rhizosphère, n'a pas été détecté dans le sol nu. La figure 6 montre, pour toutes les espèces, une baisse des effectifs, du rhizoplan vers le sol nu. Cependant ces variations sont à la limite du seuil de signification, sauf en ce qui concerne le groupe intermédiaire i2, absent au niveau du sol nu.

En intégrant données taxonomiques et pouvoir chélatant (Fig.7), il ressort que c'est essentiellement l'espèce $P$. fluorescens qui est représentée au niveau des plus fortes $\mathrm{CMI}$.

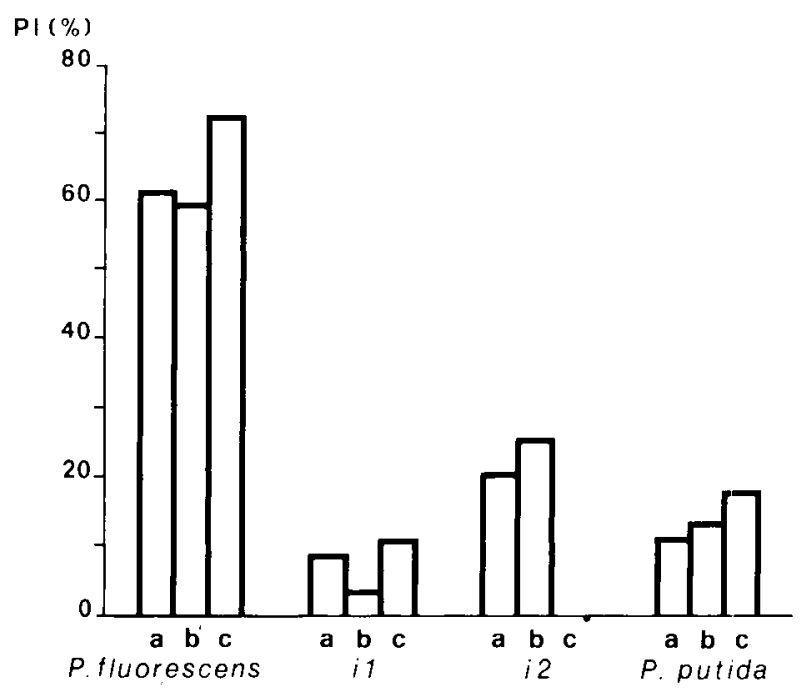

Fig.5. Répartition taxonomique des Pseudomonas selon le site d'isolement. a rhizoplan, b rhizosphère, $\mathrm{c}$ sol nu. Résultats exprimés en \% des isolats pour chaque taxon.

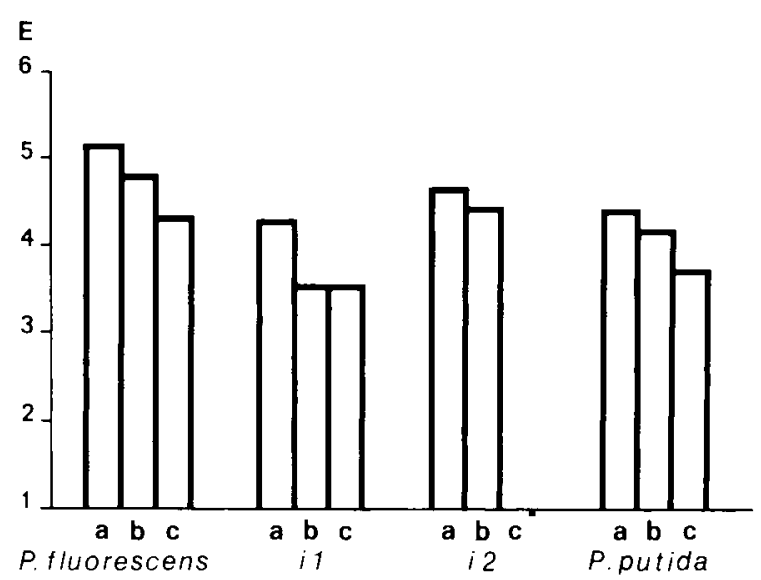

Fig. 6. Répartition taxonomique des Pseudomonas selon le site d'isolement. a rhizoplan, $b$ rhizosphère, $c$ sol nu. Résultats exprimés en effectif calculé ( $E=\log C F U / g ~ M S)$ pour chaque taxon.

\section{Discussion}

Les résultats concernant les propriétés chélatantes des Pseudomonas fluorescents vis-à-vis du fer sont à rapprocher des travaux de Lemanceau et al. (1988) sur le sol calcaire de Châteaurenard, résistant aux fusarioses. Dans les deux cas, les bactéries sont réparties selon une gamme étendue de $\mathrm{CMl}$ avec, pour le sol de truffière, apparition de 2 classes supérieures (64 et>64ppm). Compte tenu des effectifs totaux, cela suppose l'existence au niveau du rhizoplan du noisetier truffier, d'un pouvoir chélatant particulièrement puissant principalement lié aux Pseudomonas.

Les distributions diffèrent significativement selon le site d'isolement pour les deux origines géographiques (Châteaurenard et Coulaures), les bactéries du rhizoplan étant plus chélatantes que celles du sol nu. Cette différence est d'environ 10 fois dans le sol de truffière.

Ceci va dans le sens des données rapportées par différents auteurs, en particulier Reid et al. (1986), qui évaluent la concentration en sidérophores de la rhizosphère comme étant 10 à 50 fois plus élevée que celle présente dans le sol nu.

Dans le sol de truffière, les bactéries non fluorescentes représentent une forte proportion du pouvoir chélatant total. Paradoxalement leurs distributions, exprimées en effectifs, ne sont pas significativement différentes entre rhizoplan et sol nu pour les $\mathrm{CMl}$ les plus élevées ( $\geq 32 \mathrm{ppm}$ ). Les méthodes utilisées ne permettent pas de leur attribuer un rôle précis. II ne faut cependant pas rejeter une éventuelle intervention de ces germes, en particulier en cas de perturbations de l'écosystème. 

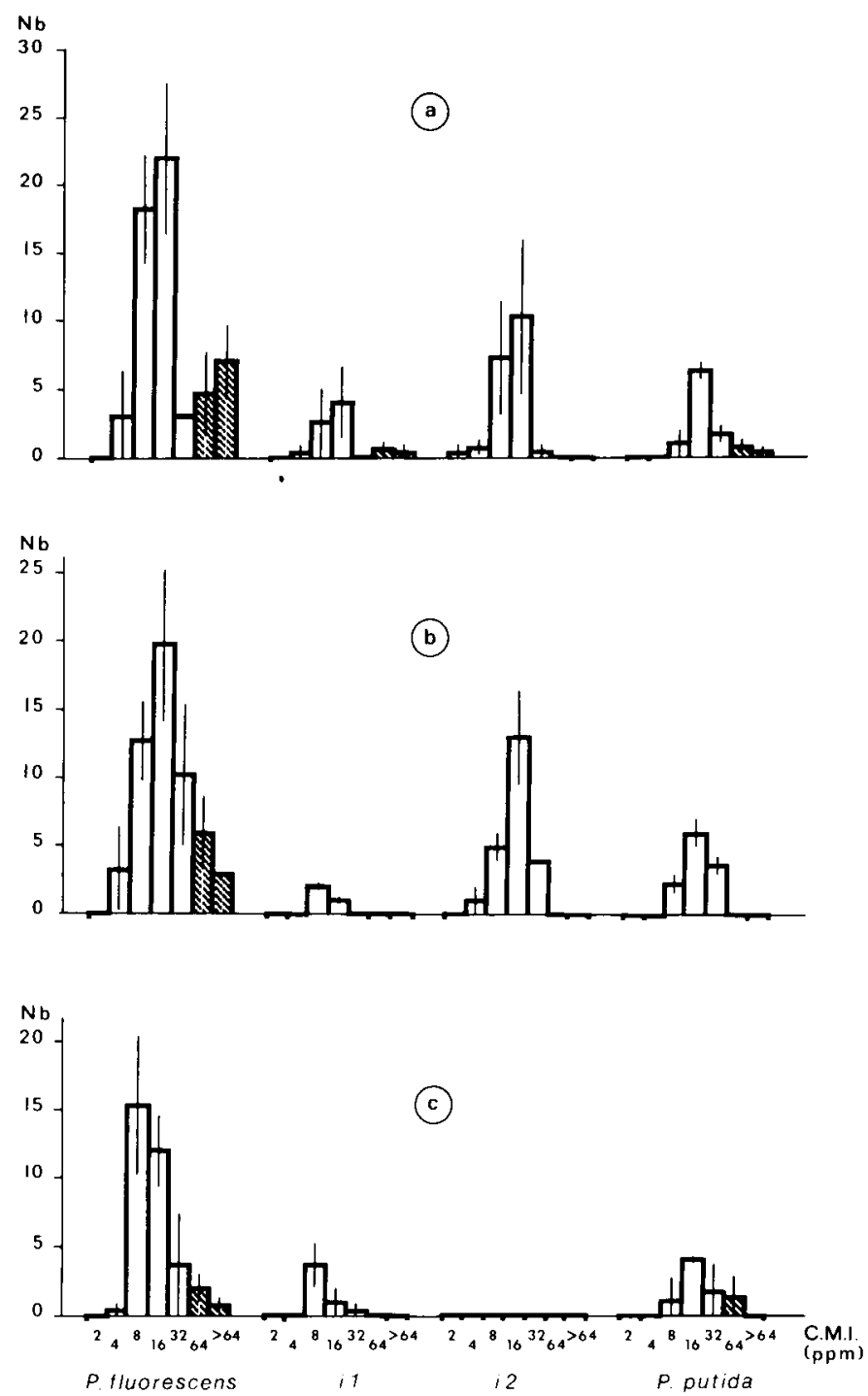

Fig. 7. Pouvoir chélatant des Pseudomonas selon le taxon et le site d'isolement. Résultats exprimés en nombre d'isolats (Nb) pour chaque concentration inhibitrice (CMI) d'hydroxy-8-quinoléine. a rhizoplan, b rhizosphère, $c$ sol nu.

Du point de vue taxonomique, les densités de $P$. fluorescens (sensu stricto) et de $P$. putida au niveau racinaire sont en accord avec les données de Vancura (1980) pour les plantes de grandes cultures. En revanche hors rhizosphère, $P$. fluorescens est plus abondant en sol de truffière $(72 \%)$ que dans les parcelles étudiées par Vancura $(50 \%)$, et l'inverse est observé avec $P$. putida (respectivement: $17 \%$ et $30 \%$ ).

En truffière, aucune différence significative liée au site d'isolement n'a été mise en évidence pour les $P$. fluorescens et putida, espèces connues pour leur implication dans différents systèmes d'antagonisme (Howell et Stipanovic, 1980; Olivier et Guillaumes, 1983; Scher, 1986; Weller et Cook, 1986).

Les effectifs indiqués (Fig. 7) sont trop faibles, quelque soit le groupe taxonomique, pour autoriser une transformation valable des résultats en effectifs calculés (E) par classe de CMI. Toutefois, les populations totales de Pseudomonas dénombrées précédemment sur le rhizoplan étant 10 fois supérieures à celles du sol nu, une transformation en effectifs réels accentuerait les différences constatées sur l'histogramme, en particulier l'importance, sur la racine, de $P$. fluorescens à pouvoir chélatant élevé. Le même raisonnement, appliqué aux $P$. putida et aux isolats appartenant au groupe i1, soulignerait leur fort pouvoir chélatant au niveau du rhizoplan. La teneur en eau du sol modifie les effectifs de Pseudomonas des sols de truffières (Olivier et Mamoun, 1988). Le potentiel hydrique influence l'équilibre Fe II/Fe III. Lemanceau et al. (1988) observent une baisse du pouvoir chélatant des Pseudomonas en fonction de l'augmentation du pF. Un déséquilibre hydrique peut entraîner une modification sélective de la population de Pseudomonas, touchant plus particulièrement un 
taxon ou certaines classes de CMI. Les conséquences sur la symbiose peuvent être de 2 ordres, soit la création de conditions défavorables compromettant l'extension de la mycorhization, soit au contraire la stimulation de populations ayant un effet bénéfique. Garbaye et Bowen (1987) avancent le concept de micro-organismes auxiliaires à la mycorhization. Mosse (1962) note, en conditions axéniques, une stimulation de l'infection endomycorhizienne du trèfle induite par un Pseudomonas. Meyer et Linderman (1986) observent, en sol non stérile, une augmentation de l'infection VA du trèfle due à un apport de $P$. putida.

II est envisageable, comme le suggère De Oliveira (1988), de favoriser l'installation et la progression "au champ" de la mycorhization établie en serre. Dans le cas de T. melanosporum, cela consisterait en une bactérisation contrôlée du plant truffier et des techniques culturales stimulant les germes utiles. Des pratiques culturales extrêmes (irrigation trop élevée, apport excessif de chélates de fer, amendement organique trop important) pourraient perturber les populations bactériennes. Les observations sur la fructification de la truffe semblent claires, mais les effets sur la mycorhization elle-même restent à déterrniner. Ce point fait l'objet du travail en cours.

\section{Remerciements}

Ce travail a bénéficié d'une aide du Conseil régional d'Aquitaine (contrat INRA-Région) et d'une subvention européenne dans le cadre du Plan d'Intégration Méditerranéenne.

\section{Références}

Ayers S.M., Rupp P. \& Johnson W.T. (1919) A study of the alkali forming bacteria in milk. Bull. US Dept. Agric., 782

Byers B.R. (1986) Siderophores and biological systems : an overview. In : Iron, Siderophores and Plant diseases. (Swinburne T.R., ed.) NATO-Plenum, Londres, pp. 1-6

De Oliveira V.L. (1988) Interactions entre les microorganismes du sol et l'établissement de la symbiose ectomycorhizienne chez le hêtre (Fagus sylvatica) avec Hebeloma crustuliniforme (Bull. ex Saint-Amans) Quél. et Paxillus involutus Batsch. ex Fr. Thèse Doct. d'Univ., Nancy-I

Frazier W.C. (1926) A method for the detection of change in gelatin due to bacteria. J. Inf. Dis. 39, 302

Garbaye J. \& Bowen G.D. (1987) Effect of different microflora on the success of ectomycorrhizal inoculation of Pinus radiata. Can. J. For. Res. 17, 941-943

Hohnadel D. \& Meyer J.M. (1986) Pyoverdine - facilitated iron uptake among fluorescent Pseudomonads. In:
Iron, Siderophores and Plant diseases. (Swinburne T.R., ed.) NATO-Plenum, Londres, pp. 119-129

Howell C.R. \& Stipanovic R.D. (1980) Suppression of Pythium ultimum - induced damping-off of cotton seedlings by Pseudomonas fluorescens and its antibiotic pyoluteorin. Phytopathology 70, 712-715

King E.D., Ward M.K. \& Raney D.E. (1954) Two simple media for the demonstration of pyocyanin and fluorescein. J. Lab. Clin. Med. 44, 301-307

Kloepper J.W., Leong J., Teintze M. \& Schroth M.N. (1980) Pseudomonads siderophores: A mechanism explaining disease - suppressive soils. Curr. Microbiol 4, 317-320

Lemanceau P. (1988) Réceptivité des sols aux fusarioses vasculaires: étude critique des théories proposées. Thèse Doct. d'Univ., Lyon-I

Lemanceau P., Samson R. \& Alabouvette C. (1988) Recherches sur la résistance des sols aux maladies. XV. Comparaison des populations de Pseudomonas fluorescents dans un sol résistant et un sol sensible aux fusarioses vasculaires. Agronomie 8, 243-249

Le Tacon F., Delmas J., Gleyze R. \& Bouchard D. (1982) Influence du régime hydrique du sol et de la fertilisation sur la fructification de la truffe noire du Périgord. (Tuber melanosporum Vitt.) dans le sud-est de la France. Acta Oecologia, Oecol. appl. 3, 4, 291306

Lindsay W.L. (1974) Role of chelation in micronutrient availability. In: The Plant Root and its Environment. (Corson E.W., ed) University Press, Charlottesville, Virginia, pp. 507-527

Luisetti J., Prunier J.P. \& Gardan L. (1972) Un milieu pour la mise en évidence de la production d'un pigment fluorescent par Pseudomonas mors-prunorum. Ann. Phytopathol. 4, 3, 295-296

Mamoun M., Poitou N. \& Olivier J.M. (1985) Etude des interactions entre Tuber melanosporum Vitt. et son environnement biotique. In: Aspects physiologiques et génétiques des mycorhizes. Actes du fer Symposium européen sur les mycorhizes, Dijon, INRA, Paris, pp. $761-765$

Meyer J.M. \& Abdallah M.A. (1978) The fluorescent pigment of Pseudomonas fluorescens: biosynthesis, purification and physiochemical properties. J. Gen. Microbiol. 107, 319-328

Meyer J.M. \& Linderman R.G. (1986) Response of subterranean clover to dual inoculation with vesiculararbuscular mycorhizal fungi and a plant growth-promoting bacterium, Pseudomonas putida. Soil Biol. Biochem. 18, 185-190

Mosse B. (1962) The establishment of vesicular-arbuscular mycorrhiza under aseptic conditions. J. gen Microbiol. 27, 509-520

Olivier J.M. \& Guillaumes J. (1976) Etude écologique des composts de champignonnières. I. Evolution de la microflore pendant l'incubation. Ann. Phytopathol. 8, 3, 283-301

Olivier J.M. \& Guillaumes J. (1983) Propriétés antagonistes des Pseudomonas fluorescents. In: Les antagonismes microbiens, $18^{e}$ colloque S.F.P. Colloq. INRA, 18, éd. INRA Publ., Versailles

Olivier J.M. \& Mamoun M. (1988) Dynamique des populations fongiques et bactériennes de la rhizosphère des noisetiers truffiers. I. Relation avec le statut hydrique du sol. Agronomie 8, 8, 711-717 
Palleroni N.J. (1984) Pseudomonadacea. In: Bergey's Manual of Systematic Bacteriology. I. Williams et Wilkins, Baltimore, Londres, pp. 141-199

Poitou N. \& Cassin C. (1987) Interaction entre le cuivre et différents champignons ectomycorhiziens comestibles. (FAL Braunschweig éd.) Mush. Sci. XII (sous presse).

Reid C.P.P., Szanislo P.J. \& Crowley D.E. (1986) Siderophore involvement in plant iron nutrition. In: Iron, Siderophores and Plant diseases, (Swinburne T.R., ed.) NATO-Plenum, Londres, pp. 29-42

Scher F.M. (1986) Biological control of Fusarium wilts by Pseudomonas putida and its enhancement by eddha. In: Iron, Siderophores and Plant diseases. (Swinburne T.R., ed.) NATO-Plenum, Londres, pp. 109-117
Sokal R. \& Rohlf J. (1969) Biometry. Chap. 16, W.H. Freeman and Co, ed.)

Szanislo P.J., Powell P.E., Reid C.P.P. \& Cline G.R. (1981) Production of hydroxamate siderophore iron chelators by ectomycorrhizal fungi. Mycologia 73, 1158-1174

Vancura V. (1980) Fluorescent Pseudomonas in the rhizosphere of plants and their relations to root exudates. Folia microbiol. 25, 168-173

Weller D.M. \& Cook R.J. (1986) Suppression of root diseases of wheat by fluorescent Pseudomonads and mechanisms of action. In: Iron, Siderophores and Plant diseases. (Swinburne T.R., éd.) NATO-Plenum, Londres, pp. 99-107 